\section{G509(P) MANAGING PARENTAL EXPECTATIONS IN PAEDIATRIC ADHD CLINICS - A NEW MODEL OF CARE}

S Perera, D Katangodage. Paediatrics, Southend University Hospital, Southend-on-Sea, UK

\subsection{6/archdischild-2015-308599.462}

Method A Prospective, questionnaire based study of parental expectations of children and adolescents attending a Paediatric ADHD follow up clinic from July 2013-august 2014. Case selection through the hospital appointment system, by parental choice.(clinician blinded).

80 completed forms were evaluated. Clinical details were verified from electronic records at data entry.

Results The age of children and adolescents who attended the clinic ranged from 6-17 years with a mean of $10.90 \mathrm{yrs}$. The M: $\mathrm{F}$ ratio was 74:6. The primary diagnoses were $\operatorname{ADHD}(43.7 \%)$, ODD (20\%), ASD (5\%), CD (5\%), LD (5\%), Tourette's, anxiety and behaviour problems. Secondary diagnoses were ADHD (25\%), ODD (15\%), Social Interaction difficulties, insomnia, anxiety and significant mental health problems

$56 \%$ were satisfied with the ADHD management, 5\% had a mixed reaction and 5\% not. $18.7 \%$ did not comment. The rest did not have ADHD.

The reasons for satisfaction were as follows 25\% happy about overall $\mathrm{Mx}, 12.5 \%$ about the medication response, 5\% about symptom improvement.no comment from the rest.

Reasons for non-satisfaction were lack of CAMHS support, lack of educational support, no improvement of symptoms.

Conclusion The model of care based on parental expectations should provide a quick and easy approach to manage the next consultations effectively by focusing on reasons for satisfactions and not during the previous. A larger study awaits.

Parental Expectations at review

\begin{tabular}{ll} 
Primary management & Secondary management \\
Aggression & Sensory issues \\
Behaviour & Sibling rivalry \\
Medication review & Non compliance \\
Symptom review & Memory improvement \\
Anxiety & Dependency on Rx \\
exclusion & housing \\
transition & DLA \\
Good Exams results & Educational support \\
Self esteem diagnosis & Hygiene counselling \\
Therapy to Stop lying & Impact of puberty \\
Therapy to Stop stealing & Relationship advice \\
Improve sleep & \\
Improve social interaction & \\
Better handwriting & \\
\hline
\end{tabular}

\section{RCPCH Quality Improvement Trainee Session}

\section{G510 IMPROVING CHILD HEALTH OUTCOMES THROUGH INTEGRATED CHILD HEALTH TRAINING CLINICS}

${ }^{1} \mathrm{C}$ Macaulay, ${ }^{2} \mathrm{~W}$ Riches, ${ }^{3} \mathrm{~J}$ Spicer, ${ }^{4} \mathrm{M}$ Lakhanpaul, ${ }^{5} \mathrm{D}$ Roland. ${ }^{1}$ Paediatrics, Evelina London Children's Hospital, London, UK; ${ }^{2}$ The Children and Young People's Health Partnership, Evelina London Children's Hospital, London, UK; ${ }^{3}$ Health Education South, London, UK; ${ }^{4}$ University College London Partners, London, UK; ${ }^{5}$ University of Nottingham, Nottingham, UK

10.1136/archdischild-2015-308599.463
Context Set in local GP Surgeries, integrated child health training clinics, for infants, children and young people under 18 years and parent/carers. The work involved secondary, community and primary care health care professionals: GP and Paediatirc Registrars, GPs, Consultant Paediatricians, Practice Nurses and Health Visitors.

Problem Child health outcomes in the UK are amongst the worst in Europe. Care is often fragmented, especially at the primary/secondary interface. The curricula across professional groups is varied and locally professionals in these pathways are unknown to each other with little shared understanding of one anothers' practices.

Assessment of problem and analysis of its causes Nationally it is reported that not all GPs have dedicated child health training. Paediatric trainees report suboptimal experience of outpatient clinics and management of long term conditions, and this was evidenced in a local survey. Integrated training was identified as a potential solution via an early pilot that suggested it could improve knowledge, skills, and working across professions. An aim was to understand the nature of this learning, if a change in outcomes was possible, and the feasibility (including economic viability) of a refined model.

Intervention A GP ST3-4 is paired with a Paediatric ST5-8 to see children together in the GP Surgery for a set of 4-6 sessions over six months. Seeing booked and "walk in" patients they are supervised locally and remotely by trainers/consultants who facilitate reflective learning. Cascading of learning happens at MDT meetings after clinics and at workshops.

Study design A pragmatic, mixed method evaluation of the pilot.

Strategy for change A representative project team led by a Registrar was set up (including a lay advocate). Workshops addressed problems and shared best practice e.g. how to get the most out of the clinics. Learning teams disseminated results locally to their peers. An aim was to develop a 'how to' guide to support roll out after the six month pilot.

Measurement of improvement Qualitative information was gathered through workshops, analysis of learning logs and interviews. Reported learning was analysed thematically. Families' feedback was achieved via questionnaires and follow up telephone interviews. A retrospective notes audit using NICE Quality Standards in four common childhood conditions was undertaken before, during and after the clinics avoiding the Hawthorne effect. A health economics model was developed to inform conclusions.

Effects of changes 44 Learning pairs and their teams were involved in learning that was rich and spanned clinical knowledge, skills and how to work well across professional boundaries. Ongoing relationship have been established with verbal handovers taking place where they did not exist before. From 848 consultations, 351 patient surveys were completed. $99 \%$ had a good experience of care with $87 \%$ more confident to manage their child. Adherence to NICE guidance moved from $57 \%$ before, to $72 \%$ during and $76 \%$ after $[\mathrm{p}<0.01]$ and suggests learning can be significant and immediate during the clinics. The economic modelling showed that only a small change in outcomes was required to make the model viable and we concluded this change was practical and achievable. This is a dynamic tool that can support integrated child health.

Lessons learnt The main barriers were clinical release for Paediatric registrars from hospital posts and embedding the MDTs. The most successful solution was getting buy-in and support from the supervisors and consultants. Once engaged, and clinics 
have started, there is almost universal support for them and increased use of the MDT.

Messages for others This project created a lot of good will locally across sectors and between health professionals. It is a viable model that can improve outcomes for children/young people though integrated training.

Please declare any conflicts of interest below This project was resourced from the local Health Education funders.

\section{G511 SAFE PRESCRIBING IN A KENYAN HOSPITAL}

L Ford. Global Links, RCPCH, London, UK

\subsection{6/archdischild-2015-308599.464}

Context This work was undertaken on the paediatric ward of a large government funded district general hospital in Kenya. The age range of the patient was from birth -12 years of age. The clinicians involved were Kenya trained Medical Officer Interns (FY-1 equivalent), Medical Officers (FY2/SHO equivalent), Clinical Officer Interns and Clinical Officers. I undertook the work in collaboration with the hospital pharmacist.

Problem Prescribing on the paediatric ward was very poor, with multiple errors. The morbidity and potential risk of mortality associated with drug errors is widely recognised. There was a lack of awareness amongst the medical staff regarding this, and the potential for harm.

Assessment of problem and analysis of its causes: I undertook a one day audit looking at the prescription charts (treatment sheets) of all inpatients on the paediatric ward. I found

Only $17 \%$ of treatment sheets were correct.

Only $4 \%$ of treatment sheets had dates indicating when the drugs had been started.

Only $2 \%$ of treatment sheets had signatures for the drugs prescribed

There were a significant number of drug errors, nearly all the errors made were prescribing the drugs which are most commonly prescribed - IV antibiotics and paracetamol.

Following discussion with the staff involved, there were a multitude of factors leading to these errors. One of the most significant was that their undergraduate education did not provide any teaching on prescribing or medication harm.

Intervention I undertook a joint teaching session on the principles of prescribing. This session had two parts - a presentation on good prescribing, and a practical prescribing scenarios. Following this session, I informed the clinicians that I would be examining the prescribing charts once a week, on an unspecified day, and on a Friday I would give a small prize to the best prescriber of that week. This is clearly at odds with how we would undertake an improvement project in the UK.

Strategy for change The main change required in this setting was a behavioural one from the prescribing clinicians following the teaching. I shared the results on the initial audit of prescribing with the staff members present at the weekly CME sessions, and formally submitted it to the head of department for paediatrics

Measurement of improvement I undertook a re-audit 4 week later using the same proforma as before. In this re-audit, $61 \%$ of treatment sheets had correct prescriptions and $61 \%$ had signatures for all medications prescribed. This was an improvement from the previous audit where on $17 \%$ of prescription sheets were correct, and only $4 \%$ at this time had signatures for all medications prescribed. There was still much room for improvement, but there was marked progress. The majority of errors in this re-audit came from a minority of clinicians.

Effects on change These changes had a significant effect on prescribing. The reduction in prescribing errors meant there was a reduced risk of harm to individual patients. The clinicians had also benefited from the education, commenting "no one has taught us to do this before", "we can use this in other departments too". One of the issues was apathy from a senior staff member who commented "its not worth it", "they'll never change".

Lessons learnt I learnt about effecting change in a low-resource setting, and the need to do this in a different manner from which I am used to. This included taking into account cultural differences within the work environment.

Message for others It is possible to effect change in this setting with simple quality improvement projects. The pharmacist has continued to deliver this teaching program, hopefully leading to sustained improvement.

No conflicts of interest.

\section{G512 MULTIDISCIPLINARY IMPLEMENTATION OF NURSE-LED PROLONGED JAUNDICE CLINIC TO IMPROVE SERVICE QUALITY AND EFFICIENCY}

${ }^{1} E$ Sage, ${ }^{2} S$ Shetty, ${ }^{3} \mathrm{M}$ Rahman. ${ }^{1}$ Paediatrics, Royal Marsden NHS Foundation Trust, London, UK; ${ }^{2}$ Paediatrics, Ashford and St. Peter's NHS Foundation Trust, Chertsey, UK; ${ }^{3}$ Paediatrics, Royal Alexandra Children's Hospital, Brighton, UK

\subsection{6/archdischild-2015-308599.465}

Context The project was undertaken in the outpatient department of a general paediatric hospital, involving paediatric junior doctors and nursing staff. Stakeholders engaged were carers and infants attending the neonatal prolonged jaundice clinic.

Problem Auditof the SHO-led prolonged jaundice clinic service showed that whilst the majority of infants were adequately screened, a large number of unnecessary and repeated blood tests and clinic follow-ups were generated; causing inefficiency of service, increased cost, and unneeded anxiety for parents.

Assessment of problem and analysis of its causes Auditof 85 infants screened and followed-up in the SHO-led prolonged jaundice clinic April 2009 to February 2010 showed that in addition to the baseline screening tests, $81 \%$ of otherwise well infants had further investigations and follow-ups performed, for which there was a clinical indication in only $20 \%$.

Presentation and in-depth discussion of audit results in the department revealed a lack of understanding of the clinic proforma and screening protocol by SHOs, leading to over-investigation with the false assumption this would aid diagnostic accuracy. The rota-system meant a different SHO was allocated to run the clinic each week, causing poor continuity and ongoing unfamiliarity with protocols.

It was proposed that an embedded nurse-led service could provide a long-term solution.

Intervention A nurse-led neonatal prolonged jaundice-clinic was created, run by four band 5 paediatric nurses with a supervisory named paediatric registrar and Consultant available during clinic if additional input was required. The nurses were trained using a 3 -stage induction programme in the clinical and operational aspects of running the clinic, and a new clinic proforma was developed. This was followed by a staged phase of observation, subsequent paediatric registrar-led supervision, and ultimately nurse-led independent practice. 